\title{
Nanosilica coating for bonding improvements to zirconia
}

This article was published in the following Dove Press journal:

International Journal of Nanomedicine

24 October 2013

Number of times this article has been viewed

\section{Chen Chen \\ Gang Chen \\ Haifeng Xie \\ Wenyong Dai \\ Feimin Zhang}

Institute of Stomatology, Nanjing

Medical University, Nanjing, Jiangsu Province, People's Republic of China
Correspondence: Feimin Zhang Institute of Stomatology, Nanjing Medical University, No I36, Han-Zhong Road, Nanjing, Jiangsu Province, People's

Republic of China 210029

$\mathrm{Tel}+86258503$ |83|

$\mathrm{Fax}+862586516414$

Emailfmzhang@njmu.edu.cn
Abstract: Resin bonding to zirconia cannot be established from standard methods that are currently utilized in conventional silica-based dental ceramics. The solution-gelatin (sol-gel) process is a well developed silica-coating technique used to modify the surface of nonsilicabased ceramics. Here, we use this technique to improve resin bonding to zirconia, which we compared to zirconia surfaces treated with alumina sandblasting and tribochemical silica coating. We used the shear bond strength test to examine the effect of the various coatings on the short-term resin bonding of zirconia. Furthermore, we employed field emission scanning electron microscopy, energy-dispersive X-ray spectroscopy, atomic force microscopy, and Fourier transform infrared spectroscopy to characterize the zirconia surfaces. Water-mist spraying was used to evaluate the durability of the coatings. To evaluate the biological safety of the experimental sol-gel silica coating, we conducted an in vitro Salmonella typhimurium reverse mutation assay (Ames mutagenicity test), cytotoxicity tests, and in vivo oral mucous membrane irritation tests. When compared to the conventional tribochemical silica coating, the experimental sol-gel silica coating provided the same shear bond strength, higher silicon contents, and better durability. Moreover, we observed no apparent mutagenicity, cytotoxicity, or irritation in this study. Therefore, the sol-gel technique represents a promising method for producing silica coatings on zirconia.

Keywords: zirconia, bond, silica coating, tribochemical silica coating, biocompatibility

\section{Introduction}

Yttria-stabilized tetragonal zirconia polycrystals (Y-TZP) have attracted great attention in dental restorations and implantations in recent years for their superior mechanical and aesthetic properties. ${ }^{1-4}$ However, inherent problems are associated with Y-TZP. Foremost, the chemical inertness of Y-TZP prevents strong and durable bond formation with conventional bisphenol-A diglycidyl dimethacrylate-based resins, while traditional mechanical and adhesive bonding to silica-based ceramics, such as acid etching and silanization, are also not applicable for the reasons above. ${ }^{5-7}$ Fortunately, silica coating, which increases the silica content and thus, the surface hydroxyls required for chemical bonding, seems to be the most promising method for promoting successful Y-TZP bonding to resin before silanization. ${ }^{8-10}$

Many methods for silica coating have been developed, which include the conventional pyrolytical silica-coated technique and the tribochemically silica-coated technique, as well as the tentative plasma spray technique and the vapor-phase deposition technique. ${ }^{11,12}$ Among these, the tribochemical silica-coating technique has reportedly improved bonding performance and offers more convenient handling than 
any other technique; therefore, it is the most commonly used method for the silica coating of high-strength ceramics. ${ }^{13-15}$ The tribochemically silica-coated technique air-abrades the bonding surface with special alumina particles that have been coated with nanosilica, embedding/coating the surface with nanosilica. This process not only provides a silica-contained surface for silanization, but it also offers micromechanical retention. However, the drawback with this technique is that sandblasting with special nanosilica-coated alumina particles results in stress on the Y-TZP surface. ${ }^{16-18}$ This has resulted in an increasingly low temperature degradation of Y-TZP, which is caused by stress-induced tetragonal-to-monoclonic phase transformations.

Therefore, a nondestructive, alternative technique for silica coating is required. A solution-gelatin (sol-gel) process for making high-purity nanosilica has been well developed, and it can mass-produce complex, dense silica components without resulting in any stress-induced processes. In our previous work, we prepared nanosilica coatings on aluminabased ceramic surfaces via the sol-gel technique and acquired encouraging results. ${ }^{19,20}$ In this study, we evaluated the effects of silica coatings prepared with our experimental sol-gel technique or with the well-used tribochemical technique on the initial bond strength of Y-TZP. Moreover, we evaluated the biological safety of the sol-gel silica coating both in vitro and in vivo.

\section{Materials and methods}

\section{Shear bond strength test}

Twenty-four pre-sintered Y-TZP blocks (KaVo Everest ${ }^{\circledR}$ ZSRonde; Kaltenbach and Voigt GmbH and Co., Bismarcking, Germany) $(12 \times 8 \times 2 \mathrm{~mm})$ were cut using a low-speed saw (IsoMet ${ }^{\circledR}$ 1000; Buehler Ltd, Lake Bluff, IL, USA), and they were then completely sintered. Twenty-four nylon tubes (inner diameter: $5 \mathrm{~mm}$; height: $2 \mathrm{~mm}$ ) were filled with a lightly-cured resin composite (Valux Plus ${ }^{\mathrm{TM}}$; $3 \mathrm{M}$ ESPE, St Paul, MN, USA), and they were lightly cured for 40 seconds (Elipar ${ }^{\mathrm{TM}}$ Freelight 2; 3M ESPE AG, Seefeld, Germany). The polymerized resin cylinders were then removed from the nylon tubes for subsequent testing.

The Y-TZP blocks were randomly assigned to three groups (eight blocks for each group) according to the following conditioning methods: Group A blocks were sandblasted (Lndp-II; Jianian Futong Medical Equipment Co., Ltd, Tianjin, People's Republic of China) with $110 \mu \mathrm{m}$ of alumina sands from a distance of $10 \mathrm{~mm}$ for 20 seconds at 3 bar pressure. Group B blocks were tribochemically sandblasted with $30 \mu \mathrm{m}$ CoJet sands (3M ESPE) from a distance of $10 \mathrm{~mm}$ for
15 seconds at 3 bar pressure. Then, silane (Porcelain Primer; Bisco, Inc., Schaumberg, IL, USA) was applied. Group C blocks were silica coated via the sol-gel technique according to the following steps: a layer of silica film was formed through brush-coating of the nanosilica solution (prepared using alkoxide hydrolysis of tetraethyl orthosilicate with $\mathrm{HCl}$ as a catalyst). A drop of hydroxide was then added, which was air-dried for a few seconds before thermal treatment to $400^{\circ} \mathrm{C}$. Then, silane (Porcelain Primer; Bisco, Inc.) was applied to the surface.

A resin-composite cylinder was cemented onto the treated Y-TZP block with bisphenol-A diglycidyl dimethacrylate composite resin cement (Choice; Bisco, Inc.). Shear bond strength (SBS) was analyzed with a universal testing machine (ElectroPuls $^{\mathrm{TM}} 3365$ Instron $^{\circledR}$, Norwood, MA, USA) using a crosshead speed of $1.0 \mathrm{~mm} /$ minute after 24 hours of storage in distilled water at room temperature. One-way analysis of variance (ANOVA) and the least significant difference test for multiple comparisons were used for statistical analysis. The statistical software package used was the Statistical Package for the Social Sciences version 11.5 (IBM Corporation, Armonk, NY, USA) ( $\alpha=0.01)$.

\section{Surface characterization and water-mist characterization testing}

Micromorphological characterization

Transmission electron microscopy (JEM-2100; JEOL, Tokyo, Japan) was used to observe the nanosilica solution. Field emission scanning electron microscopy (FESEM) (LEO-1530VP; LEO Elektronenmikroskopie GmbH, Oberkochen, Germany), in the secondary or backscatter modes, was used to characterize Y-TZP surfaces treated with alumina sandblasting, tribochemical silica coating, and solgel silica coating. In order to compare the differences between the alumina-sandblasted surface and the silica-coated surface in one backscattered scanning electron microscopy (SEM) field, the Y-TZP block was alumina-sandblasted first and then tribochemically silica-coated or sol-gel silica-coated on parts of the alumina-sandblasted surface.

\section{Water-mist damage testing}

Y-TZP blocks treated with tribochemical silica coating or sol-gel silica coating were subjected to damage testing, conducted by water-mist spraying (30 seconds and 5 minutes) via a pressure spray gun on a dental chair unit (Performer ${ }^{\circledR}$; A-dec, Inc., Newberg, OR, USA) at 3 bar pressure. All the blocks were then dried, gold-sputtered, and examined by FESEM. 


\section{Energy-dispersive $\mathrm{X}$-ray spectroscopy analysis}

Energy-dispersive X-ray spectroscopy (EDS) analysis (INCAx-sight; Oxford Instruments, Oxfordshire, UK) was used for characterizing the alumina-sandblasted Y-TZP surface, the tribochemically silica-coated Y-TZP surface, and the sol-gel silica-coated Y-TZP surface with or without watermist damage. These were performed under the same magnification, with a detection area of approximately $40 \times 100 \mu \mathrm{m}^{2}$. The EDS scanning was repeated three times.

\section{Atomic force microscopy}

Atomic force microscopy (AFM) (PicoPlus; AstraZenca, London, UK) in contact mode was used to evaluate the structure and surface roughness of the sol-gel nanosilica coating. Imaging was conducted in air at room temperature using a standard silicon (Si) tip.

\section{Fourier transform infrared spectroscopy}

The untreated Y-TZP surface and the sol-gel silica-coated Y-TZP surface were examined by Fourier transform infrared (FTIR) spectroscopy in reflection mode (Nexus ${ }^{\circledR} 870$; Thermo Electron Corporation, Madison, WI, USA).

\section{Salmonella typhimurium reverse mutation assay (Ames mutagenicity test)}

Histidine-requiring Salmonella typhimurium strains TA97, TA98, TA100, and TA102 were chosen to test the mutagenicity of the silica coatings. Bacteria were exposed to defined conditions of various doses of testing standard leach liquors, and they were incubated for 48 hours. Standard leach liquor was prepared with silica coating from the sol-gel technique and normal saline (NS) under the ratio of $0.2 \mathrm{~g} / \mathrm{mL}$ (mass/volume) at $37^{\circ} \mathrm{C}$ for 24 hours. Four different doses of standard leach liquor were tested in this study $(400 \mu \mathrm{L}$, $200 \mu \mathrm{L}, 100 \mu \mathrm{L}$, and $50 \mu \mathrm{L}$ per plate). Distilled water and dimethyl sulfoxide were chosen as negative controls, while 2-aminofluorene, chrysazin, dexon, and sodium azide were used as positive controls. The number of mutant colonies with or without S9 (liver fractions of rats used for enzyme induction) was investigated after a 48-hour incubation period, and the results were subject to statistical analysis. The procedure details followed the medicine standards of the People's Republic of China (YY/T 0127.10-2009).

\section{Cytotoxicity test}

Standard leach liquor was prepared as mentioned previously. Leach liquor (100\%) was chosen as the testing dose and NS as the negative control. The fibroblast cell line L-929
(NCTC clone 929: CCL 1) was chosen for this test. The agar diffusion test was adopted to evaluate the cytotoxicity of silica coating. The details of the test procedures were in accordance with the medicine standards of the People's Republic of China (YY/T 0127.9-2009). Briefly, the cells were cultured until they reached the end of the logarithmic growth phase. Then, $10 \mathrm{~mL}$ of cell suspension $\left(2.5 \times 10^{5}\right.$ cells $\left./ \mathrm{mL}\right)$ was pipetted into a Petri dish and incubated at $37^{\circ} \mathrm{C} \pm 2^{\circ} \mathrm{C}$ in a water-saturated atmosphere with 5\% (volume fraction) carbon dioxide for 24 hours. L-929 was covered with a freshly prepared agar/ culture medium mixture. The agar/culture medium mixture was allowed to solidify at room temperature (approximately 30 minutes). Then, $10 \mathrm{~mL}$ of neutral red solution was added to the dish and kept dark for 15-20 minutes, and then excess neutral red solution was aspirated. Round filter papers with a diameter of $5 \mathrm{~mm}$ were soaked with $0.01 \mathrm{~mL}$ of test leach liquor or NS and added to the surface of the agar plates. Decolorization zones around the filter papers were assessed after 24 hours, and the decolorization and lysis indices for each test sample were determined.

\section{Oral mucous membrane irritation test}

The oral mucous membrane irritation test was conducted in accordance with the medicine standards of the People's Republic of China (YY/T 0127.13-2009). Three adult female Syrian hamsters were chosen for this test. Standard leach liquor was prepared as above. Leach liquor (100\%) was the only testing dose, while NS was used as the negative control. Leach liquor-infiltrated cotton-wool pellets were placed in one hamster cheek pouch, while NS-infiltrated pellets were placed in the contralateral side. The animals were sacrificed after 15 days. The pouches were examined macroscopically following removal of the pellets. The appearance of the cheek pouches for each animal was analyzed, and the pouch surface reactions for erythema were graded according to the standard. Tissue samples from representative areas of the pouches were removed for histological examination. Hematoxylin and eosin staining was performed to evaluate irritation. A pathologist evaluated the microscopic irritant effects from the oral tissues. The pathologist graded each tissue sample relative to the standard. Moreover, the weight of each animal was recorded every day and if it decreased continuously, the particular hamster was weeded out.

\section{Results}

\section{Shear bond strength test}

Initial SBS values of the three groups are shown in Figure 1. One-way ANOVA revealed significant differences in the SBS 


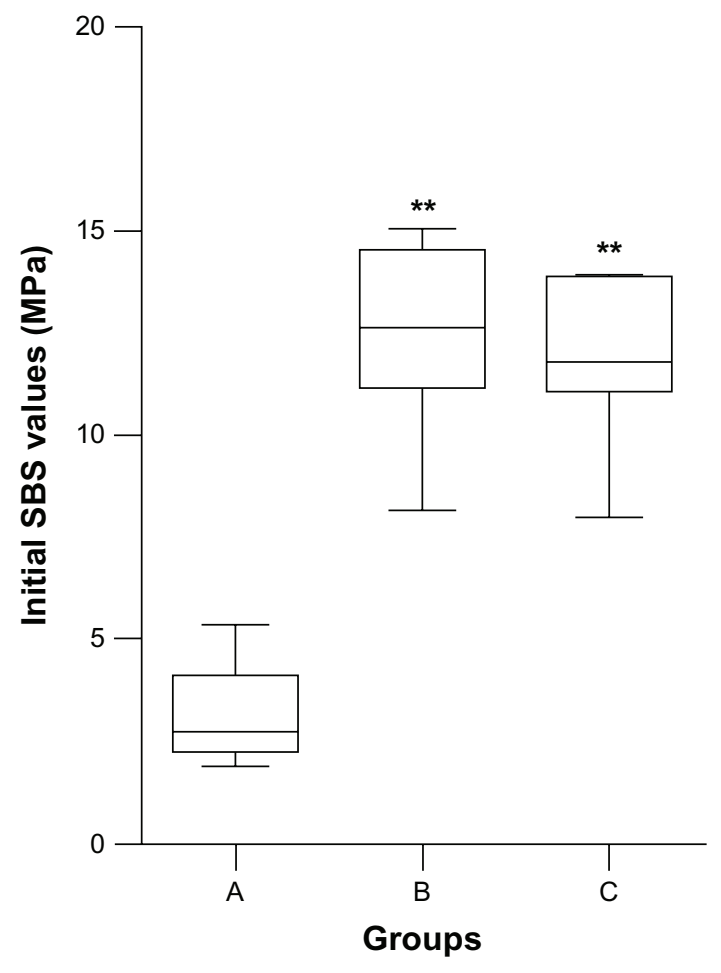

Figure I Boxplot of initial SBS values for the alumina-sandblasting, tribochemical silica-coating and sol-gel silica coating groups.

Note: **Statistical difference compared with group A $(P<0.01)$; no statistical difference between group $B$ and group $C$.

Abbreviations: SBS, shear bond strength; sol-gel, solution-gelatin. values for the experimental groups, depending on the surface treatment factors $(F=62.354 ; P=0.000)$. According to results of the least significant difference tests, group A showed lower SBS values than both group B $(P=0.000)$ and group $\mathrm{C}(P=0.000)$; the other two groups showed higher values. Moreover, no statistical differences were detected between group $\mathrm{B}$ and group $\mathrm{C}(P=0.613)$.

\section{Surface characterization and water-mist damage testing \\ Micromorphological characterization}

Nanosized silica particles were observed in the transmission electron microscopy images (Figure 2A). FESEM images showed that the Y-TZP surface sandblasted with alumina sands had a roughened texture and a clear grain structure (Figure 2B). Meanwhile, the tribochemically silica-coated Y-TZP surface presented a similar roughened morphology compared to the alumina-sandblasted one; a large number of nanoparticles were observed on the intergrains (Figure 2C). The lower magnified backscattered SEM images presented no significant differences between the components of the tribochemically-sandblasted regions and the alumina-sandblasted regions (Figure 2D), while a perceptible figure in
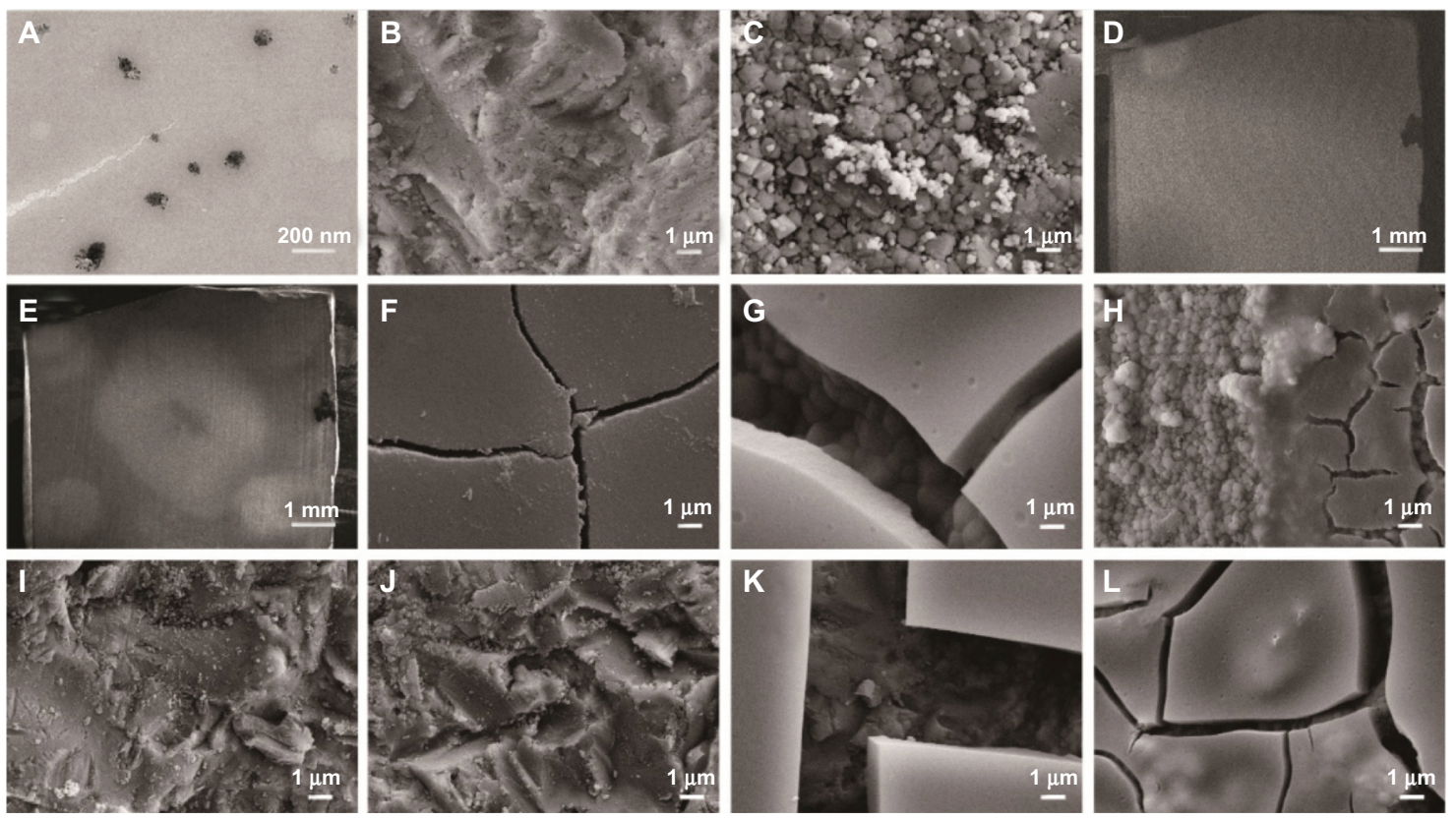

Figure 2 TEM image of silica sol and FESEM images of Y-TZP surfaces treated from different processes.

Notes: TEM image of (A) silica sol (200 KV); (B) Y-TZP treated with alumina sandblasting; and (C) Y-TZP treated with tribochemical silica coating. (D and E) represent the backscattered image and second electronic image of tribochemically silica-coated surfaces at low magnification, respectively. (F) Y-TZP treated with sol-gel silica coating before thermal treatment and $(\mathbf{G})$ after thermal treatment. $(\mathbf{H})$ Comparison of the sol-gel silica-coated surface and the alumina-sandblasted surface under backscattered mode. Moreover, ( $\mathbf{I}$ and $\mathbf{J}$ ) show the tribochemically silica-coated surface subjected to water-mist spraying for 30 seconds and 5 minutes, respectively. In addition, (K) and (L) present the sol-gel silica-coated surface subject to water-mist spraying for 30 seconds and 5 minutes, respectively.

Abbreviations: TEM, transmission electron microscopy; sol, solution; FESEM, field emission scanning electron microscopy; Y-TZP, yttria-stabilized tetragonal zirconia polycrystals; sol-gel, solution-gelatin. 
the tribochemically-sandblasted region was observed in the second SEM image (Figure 2E). On the sol-gel silica-coated Y-TZP surface prior to baking, smooth micromorphology was observed (Figure 2F), while after thermal treatment, obvious cracks on the coating were observed (Figure $2 \mathrm{G}$ ). Moreover, in the backscattered FESEM image, significant differences can be seen along the boundary of the sol-gel silica-coated surface and the alumina-sandblasted surface (Figure 2H).

After water-mist spraying for different times, a decreased number of nanoparticles were observed in the FESEM images (Figure 2I and J) for the tribochemically silica-coated surface (Figure 2C). Moreover, fewer nanoparticles were observed in Figure 2J compared to Figure 2I, which suggests that water-mist spraying for longer periods of time removed more of the nanoparticles that adhered to the Y-TZP surface. However, FESEM observations revealed water-mist spraying had less of an effect on the sol-gel silica-coated surface (Figure $2 \mathrm{~K}$ and $\mathrm{L}$ ).

\section{EDS analysis}

$\mathrm{Si}$ and aluminum elements in the tribochemically silicacoated surface were detected from EDS mapping (Figure 3A). The distribution areas were identical and they appeared to be pointed in shape, reflecting the points of impact with the CoJet sands. Lower brightness indicates lower Si content. Si elements were predominately detected in the EDS mapping of the sol-gel silica-coated surface, which suggests that the Si elements in the sol-gel coating covered the zirconium (Zr) elements. Accordingly, EDS mapping only detected Zr

A

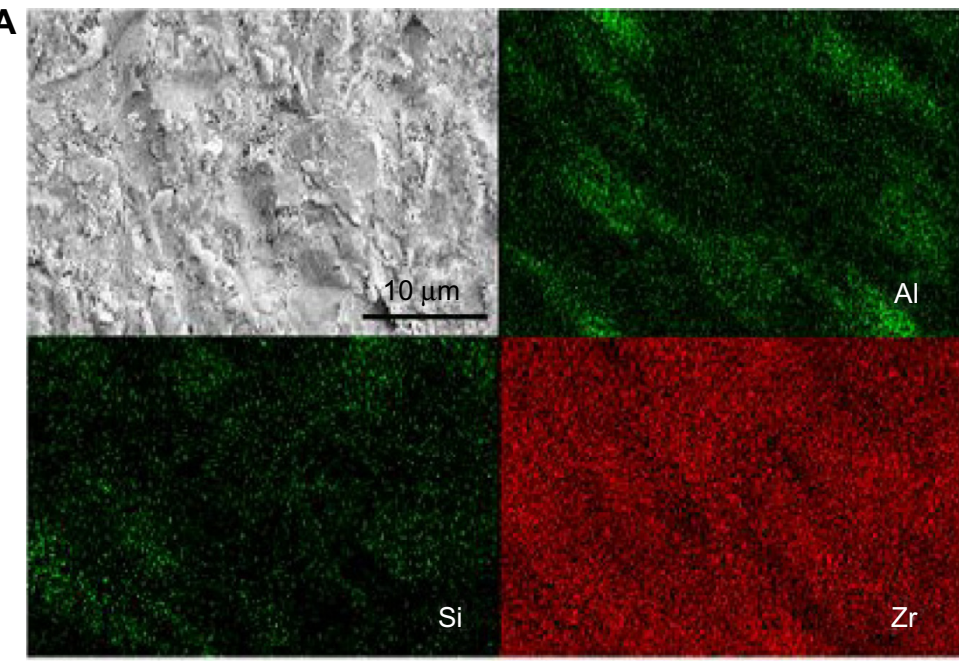

B
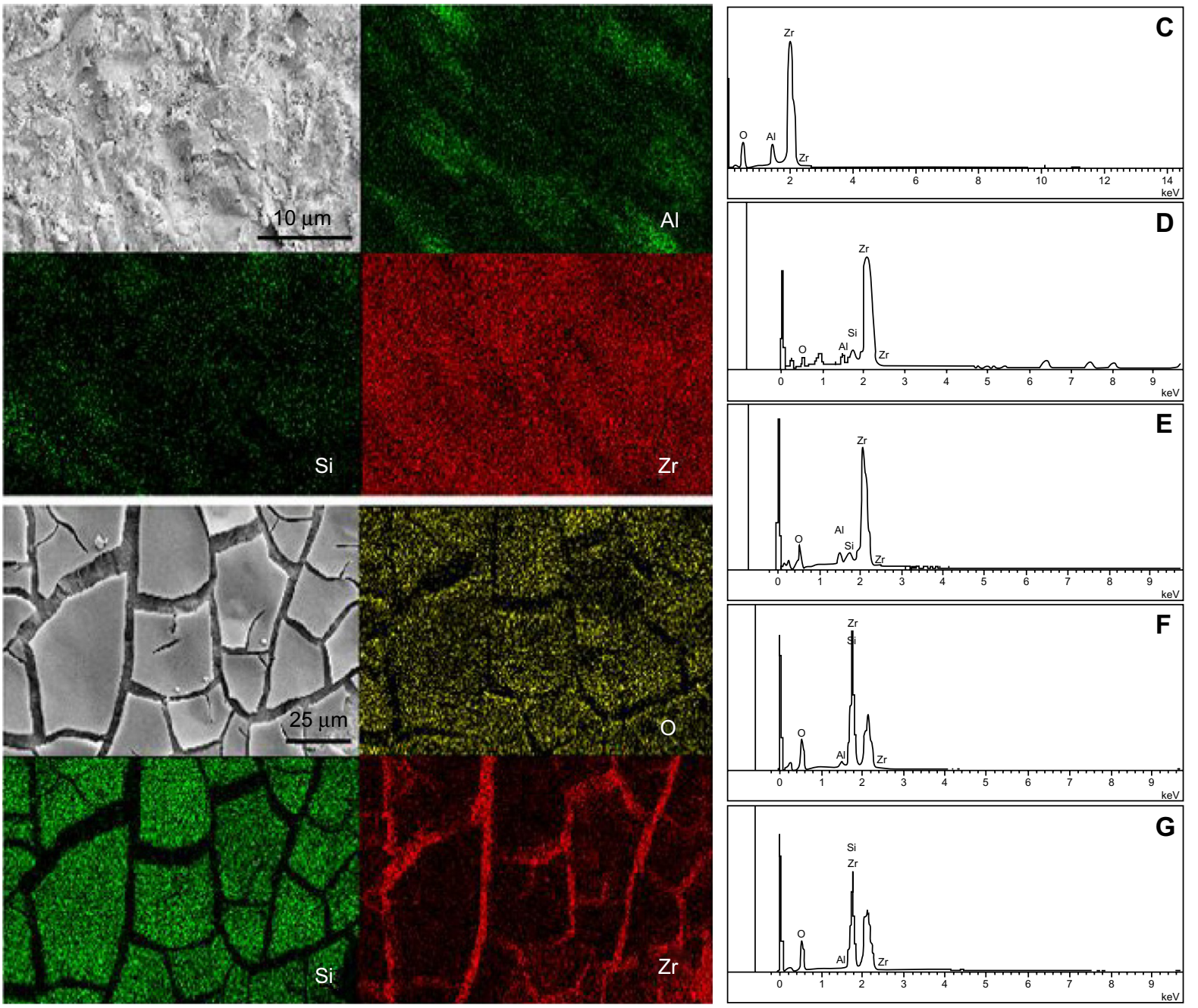

Figure 3 EDS mapping images of tribochemically silica-coated and sol-gel silica-coated Y-TZP surfaces.

Notes: EDS mapping images of $(\mathbf{A})$ tribochemically silica-coated and $(\mathbf{B})$ sol-gel silica-coated $Y$-TZP surfaces. (C-G) EDS spectra of Y-TZP surfaces treated with different processes. (C) Y-TZP treated with alumina sandblasting; (D and E) show a tribochemically silica-coated surface subjected to water-mist spraying for 30 seconds and 5 minutes, respectively. Finally, (F and $\mathbf{G}$ ) show a sol-gel silica-coated surface subjected to water-mist spraying for 30 seconds and 5 minutes, respectively.

Abbreviations: Al, aluminum; Si, silicon; EDS, energy-dispersive X-ray spectroscopy; sol-gel, solution-gelatin; Y-TZP, yttria-stabilized tetragonal zirconia polycrystals; $\mathrm{Zr}$, zirconium; O, oxygen. 
elements within the cracks among the coating (Figure 3B). No Si elements were found in the EDS spectrum of the alumina-sandblasted surface (Figure 3C).

EDS spectra showed a decrease in Si elements (from $2.4 \mathrm{wt} \%$ to $1.9 \mathrm{wt} \%$ ) for the tribochemically silica-coated surface after water-mist spraying (Figure 3D and E). A decrease in Si levels was also detected for the sol-gel silica-coated surface after water-mist spraying; however, overall Si levels were much higher (45.6 wt\% after 30 seconds of spraying; $27.8 \mathrm{wt} \%$ after 5 minutes of spraying) (Figure 3F and G).

\section{AFM examination}

AFM images (Figure 4) showed that the sol-gel silica coating consisted of cross-linked nanoparticles, and the root mean square roughness of the coating was $252.93 \pm 64.97 \mathrm{~nm}$.

\section{FTIR analysis}

The FTIR spectra for the Y-TZP surface before and after coating with sol-gel silica are presented in Figure 5. The strong peaks at $1,091 \mathrm{~cm}^{-1}$ and $1,061 \mathrm{~cm}^{-1}$ were attributed to the vibrations of the $\mathrm{Si}-\mathrm{O}-\mathrm{Si}$ groups. The $961 \mathrm{~cm}^{-1}$ peak was ascribed to $\mathrm{Si}-\mathrm{OH}$ groups.

\section{Ames mutagenicity test}

Test samples were considered mutagenic if the number of mutant colonies increased by more than two times when compared to the negative control, either in a dose-dependent manner; if just one dose was used, the same result was reproduced. We detected clear and normal bacterial lawns formed by microcolonies in both the test and control groups. No increase in the number of mutant colonies was detected under four different doses of standard leach liquor, compared to the negative control. However, for the positive control, more than twice the number of mutant colonies was detected compared to the negative control. No statistical differences were presented with or without S9 (Figure 6).

\section{Cytotoxicity test}

We used an inverted microscope to observe cells cultured in the test and control groups. Both groups showed a spindlelike shape with a clear border and intact cell membranes. The assessments for the decolorization index and the lysis index were in accordance with the standard. Both of the indices for the $100 \%$ leach liquor group and the control group were 0 , indicating test samples were not cytotoxic (Figure 7).

\section{Oral mucous membrane irritation test}

Grades for each macroscopic observation were added together and the sum was divided by the number of observations to determine an average grade per animal. A total score of more than nine (determined from microscopic evaluation of the cheek pouches of controls) indicated underlying pathology. The grades for all animals in the test group were
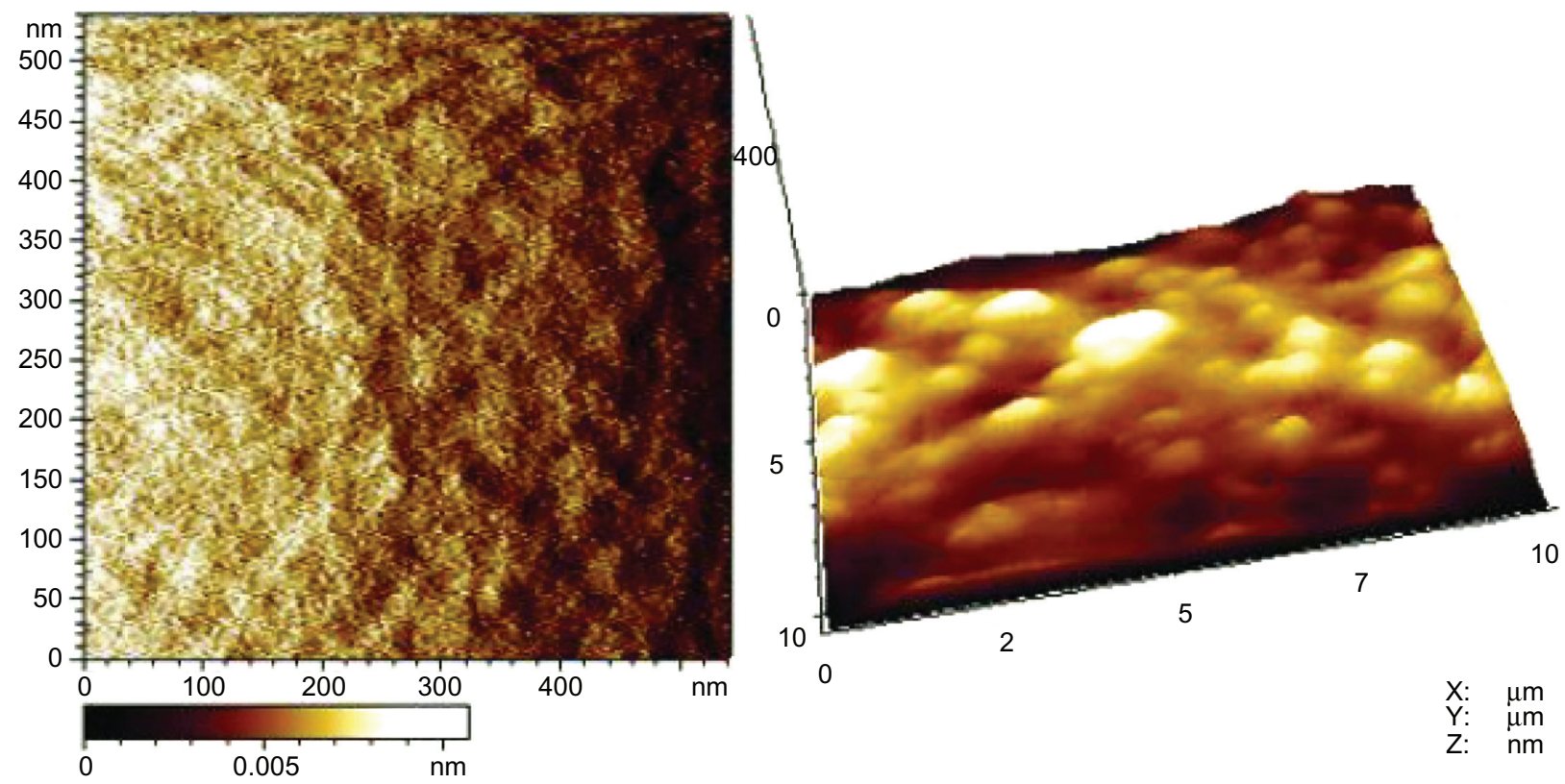

Figure 4 AFM images of sol-gel silica coating.

Note: Left, spot size of $500 \times 500 \mathrm{~nm}^{2}$; right, spot size of $400 \times 10 \mu \mathrm{m}^{2}$.

Abbreviations: AFM, atomic force microscopy; sol-gel, solution-gelatin. 


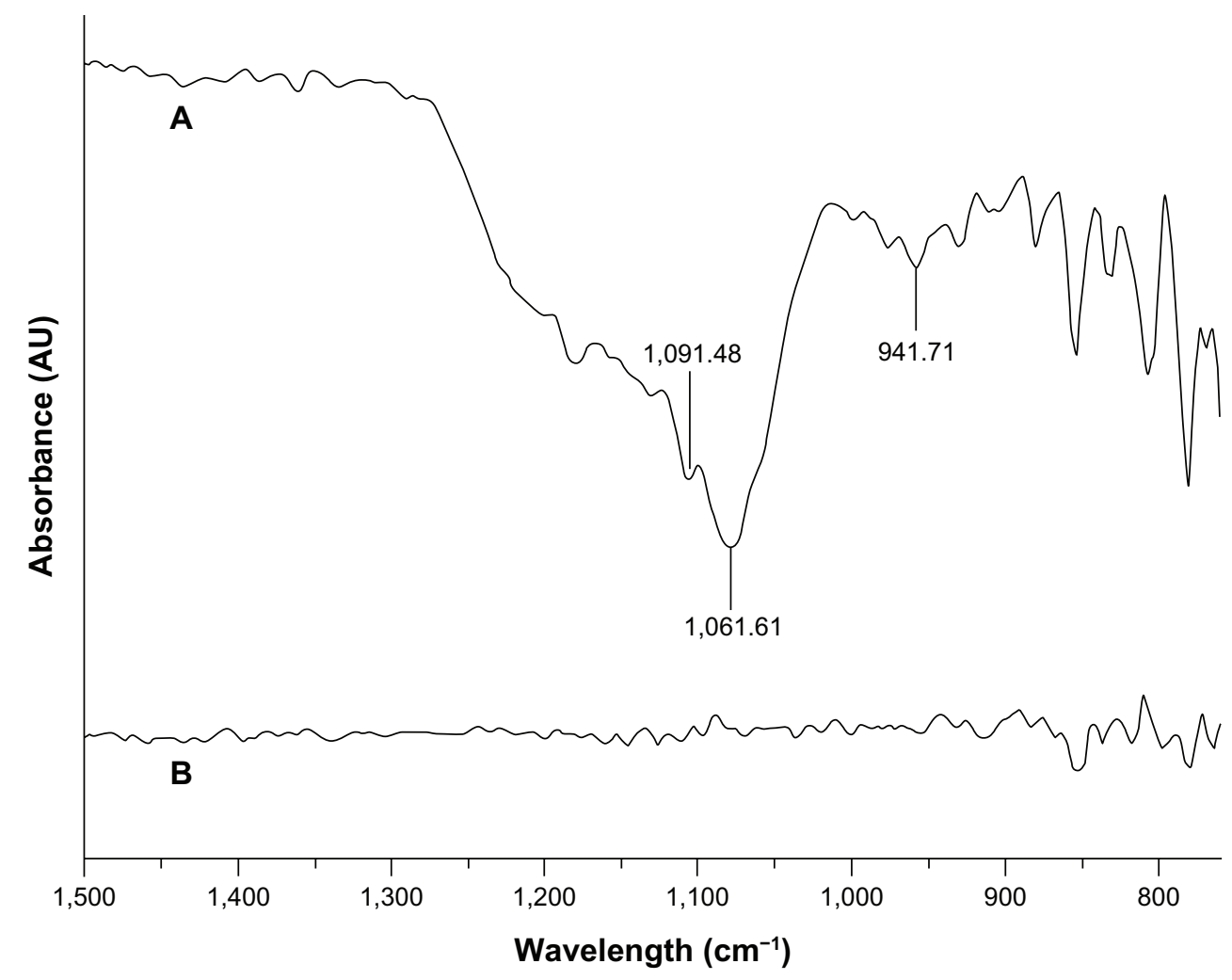

Figure 5 FTIR spectra of the untreated Y-TZP surface and the sol-gel silica-coated Y-TZP surface.

Note: (A) Sol-gel silica-coated Y-TZP surface; (B) untreated Y-TZP surface.

Abbreviations: FTIR, Fourier transform infrared; Y-TZP, yttria-stabilized tetragonal zirconia polycrystals; sol-gel, solution-gelatin.

then added together and the sum was divided by the number of observations to obtain a test group average. The control group average was subtracted from the test group average to yield the irritation index.

The weights of the hamsters did not change statistically throughout the 15 days. The scores for the macroscopic observations for the testing side and contralateral side were both 0 , indicating that the silica coating did not cause any irritation. Furthermore, the scores from the microscopic evaluation for both sides were 0.67 , indicating no apparent irritation reaction. Figure 8 shows representative images from histological sections of both cheek sides.

\section{Discussion}

The sol-gel process is one of the most useful and versatile methods for oxide film fabrication due to the low processing temperature, homogeneity of coatings, easy control of coating thickness, and the potential to produce coatings on solids with complex shapes. In this study, we used acid-catalyzed alkoxide-hydrolysis to prepare silica sol with nanosized particles (shown in Figure 2A). To establish whether our sol-gel coating would improve the bonding of Y-TZP, we compared it to alumina-sandblasting and tribochemical silica coating. We observed higher SBS in both silica-coated groups compared to the alumina-sandblasted group. The FESEM images revealed a smooth surface for the sol-gel silica coating, which was compared to the roughened texture observed for the alumina-sandblasted and tribochemically silica-coated surfaces. EDS mapping predominately detected Si elements and trace $\mathrm{Zr}$ elements on this surface, which suggests that the Y-TZP surface was totally replaced by a new smooth silica-coating surface. Characteristic peaks for silica appeared in the FTIR spectrum of the solgel silica-coated Y-TZP surface, which also illustrates the conversion. AFM images revealed that the sol-gel silica coating contained homogeneously cross-linked particles and presented small root mean square roughness. Obviously, such small roughness of the sol-gel silica-coated Y-TZP surface could not provide the microinterlock for resin bonding. No Si elements were found in the EDS spectrum of the alumina-sandblasted surface, while EDS detected increased Si elements for the tribochemically silica-coated surface. FESEM images showed that the difference between the two surfaces was that the former presented a large number of nanoparticles. According to the EDS mapping, the distribution areas of $\mathrm{Si}$ and aluminum elements in the 
A

D
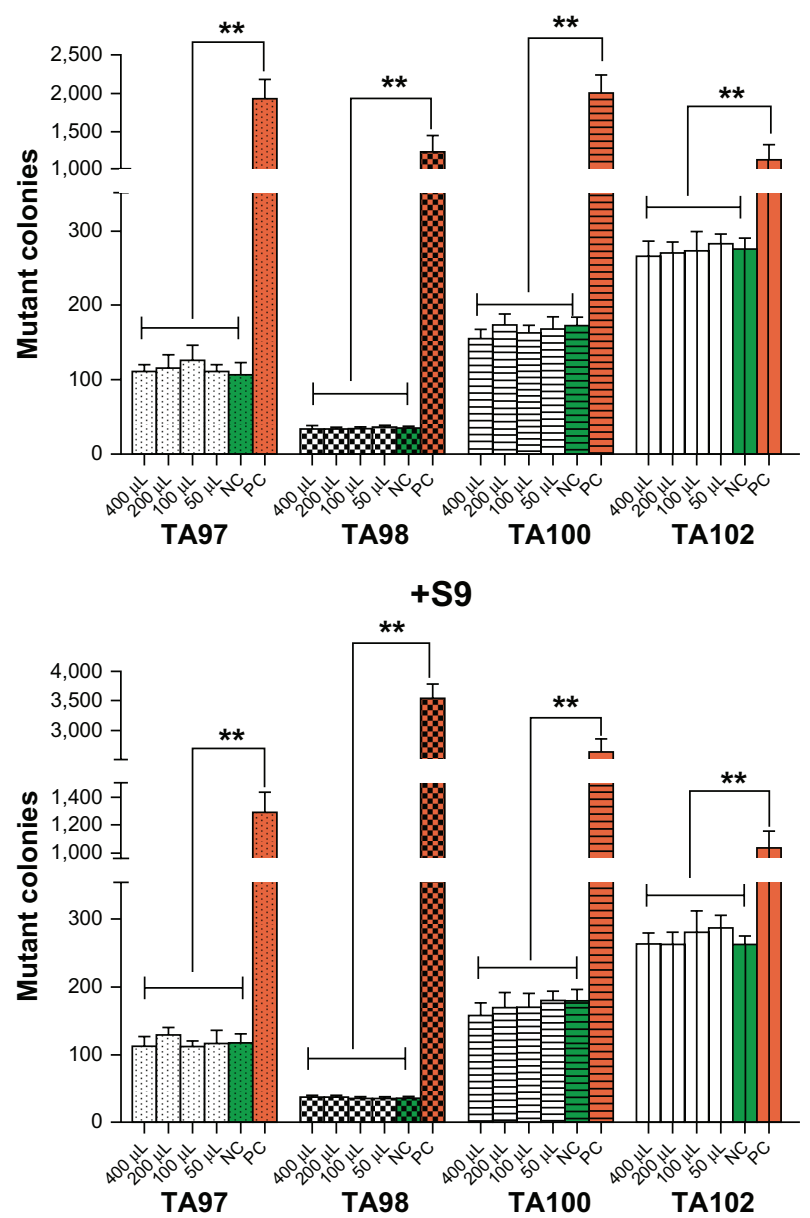

Figure 6 Representative photomicrographs of mutant colonies from the Ames test. Notes: (A-C) Representative photomicrographs of mutant colonies from the Ames test. (A) PC; (B) NC; (C) leach liquor of silica coating; (D) statistical results. **The number of mutant colonies of PC is above twice that of the other groups. Abbreviations: S9, liver fractions of rats used for enzyme induction; NC, negative control; PC, positive control.

tribochemically silica-coated surface were identical and appeared to be pointed in shape, reflecting the points of impact with the CoJet sands. Therefore, these nanoparticles on the tribochemically silica-coated surface should be nanosilica particles that have come from the special CoJet sands. In line with previous findings, we speculate that the increased hydroxyl groups resulting from silica and the subsequent silanization contributes to the improved bonding of both the tribochemically silica-coated surface and the sol-gel silica-coated surface, ${ }^{8,921,22}$ while the microinterlock does not seem to be as important.

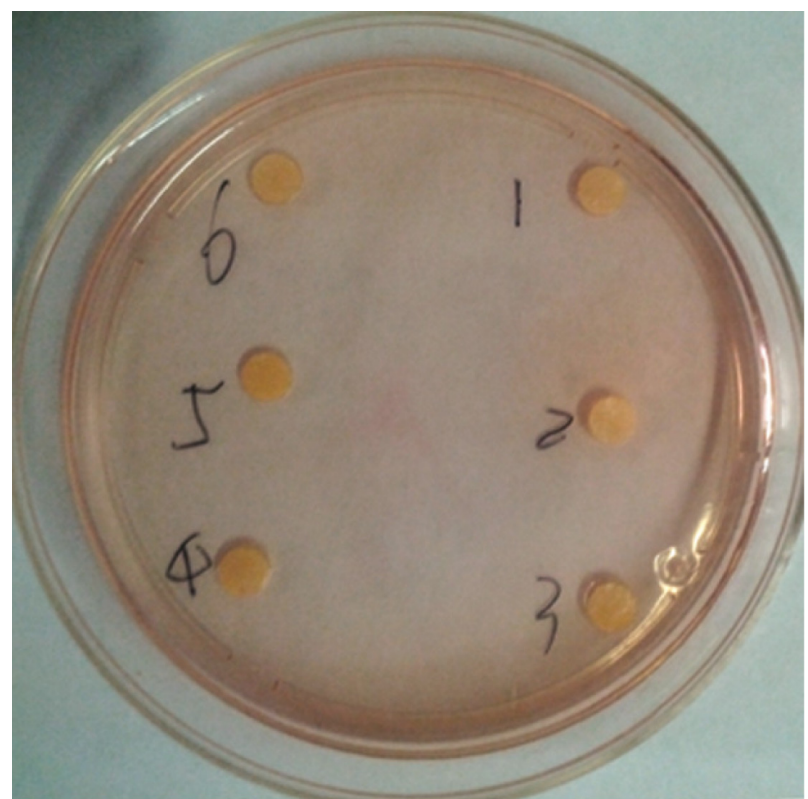

Figure 7 Agar diffusion test for cytotoxicity.

Note: Numbers I-3 represent the leach liquor of the silica coating; numbers 4-6 represent NS.

Abbreviation: NS, normal saline.

As the solvent evaporated, the silica sol converted to a gel on the Y-TZP surface. According to previous studies, the nanosilica gel coating attaches to ceramic surfaces by van der Waals electrostatic interaction forces, and the $\mathrm{Si}-\mathrm{O}-\mathrm{Si}$ network is strengthened by baking. ${ }^{23}$ In the present study, the sol-gel silica coating was smooth and continuous prior to thermal treatment; however, after baking, many deep cracks formed on the Y-TZP surface, produced assumingly by heat shrink stress. Fortunately, no peel-off was observed and the cracks did not seem to influence resin/Y-TZP bonding. Even so, controlling the quality of coating with low cracking is presumably necessary, through using some methods (such as slowing down the heating rate), since shrink stress produced during the thermal treatment would theoretically weaken the strength of the nanosilica gel coating and the bonding between the nanosilica gel coating and Y-TZP.
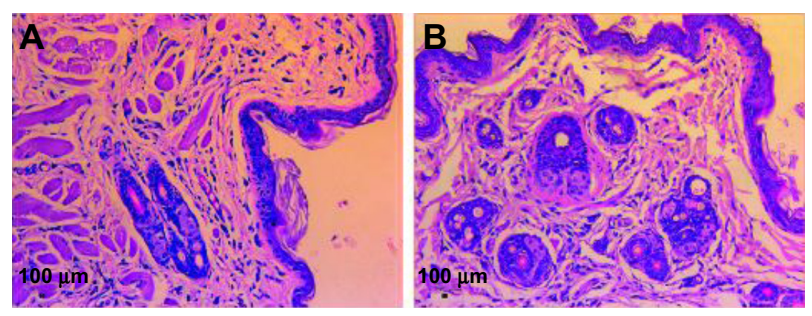

Figure 8 Representative images of the histologic section stained by hematoxylin and eosin.

Notes: (A) Normal saline group; (B) leach liquor group. 
An intriguing result from this study is that improved bonding was observed for both the sol-gel silica coating and the tribochemical silica-coating surfaces. Albeit, the overall Si content was higher for the sol-gel silica coating compared to the tribochemical technique. Moreover, backscattered SEM images presented a drastic contrast between the sol-gel silica coating surface and the alumina-sandblasted surface, while no significant differences were observed between the tribochemical silica-coating surface and the alumina-sandblasted surface, suggesting that the increased Si elements from the sol-gel nanosilica coating surface are advantageous when compared to the tribochemical nanosilica coating surface. Unexpectedly, the sol-gel silica surface did not produce higher SBS; the reasons for this will require further investigation. Nevertheless, the sol-gel technique proves to be a promising method in improving resin bonding to zirconia by avoiding the potential damage to the mechanical properties brought on by sandblasting.

Water-mist spraying is a convenient and commonly used method in clinics for cleaning the bonding surfaces of prostheses. Spraying usually lasts from several seconds to tens of seconds, depending on the handling habits of the dentists. In the present study, in order to evaluate the influence watermist spraying has on the silica coating, a representative 30 seconds of spraying time was adopted to simulate handling in clinics, and a prolonged 5-minute spraying time was adopted to evaluate the durability of the silica coating to more drastic water-mist spraying. Since longer spraying time is not employed in clinics, we did not conduct water-mist spraying experiments for anything greater than 5 minutes. Chen et $\mathrm{al}^{24}$ previously reported that silica particles adhered to the Y-TZP surface from tribochemical silica coating, and they were removed by forceful water stream, which inducing decreased resin bonding. Similarly, Nishigawa et $\mathrm{al}^{21}$ reported that ultrasonic cleaning decreased the silica content on the tribochemically silica-coated surfaces and this, therefore, decreased the adhesion efficacy of Y-TZP to resin. In the present study, we also observed a similar result, as water-mist spraying decreased the number of nanoparticles on the tribochemically silica-coated surfaces. Fortunately, however, according to our SEM/EDS analysis, the sol-gel silica-coated surface demonstrated better durability against spraying damage compared to the tribochemically silicacoated surface.

Besides bonding performance, biocompatibility is also an important factor to be considered for silica-coating surfaces, especially in consideration of the long service time of these restorations in intraoral environments.
S. typhimurium histidine-required strains cannot synthesize histidine, which is necessary for growth. Therefore, exposure to genotoxic agents in test samples may induce mutations within specific histidine-required genes, enabling them to synthesize histidine, and subsequently allowing them to survive in environments that are deprived of histidine (wild type). Such induction causes a dose-related increase in the numbers of mutant colonies. In our study, the silica coating did not show apparent toxicity to the strains, as demonstrated by clear and normal bacteria lawns formed by microcolonies. No mutagenicity was observed in any of the test groups.

Compared to animal assays, cell culture is more sensitive to cytotoxic agents. The agar diffusion test provides useful information to aid in predicting the potential clinical applications in humans. Cells in good condition can absorb neutral red while cultured in the agar diffusion test, while cytotoxic agents (which are diffused through agar) can disrupt this, causing decolorization and lysis. According to our results, the nanosilica coating prepared by the sol-gel technique presented little effect on cell growth. In vivo irritation tests are critical to medical devices that are used orally in the long term. We observed no macroscopic or microscopic pathological changes.

\section{Conclusion}

Based on the present study, and within its limitations, the following conclusions might be drawn:

1. The combination of silica coating and silanization may improve the initial bond strength of Y-TZP. The increased hydroxyl groups from the silica and its subsequent silanization, rather than microinterlock, ultimately contributed to the improved bonding of both the tribochemically silica-coated surface and the sol-gel silica coated surface.

2. Sol-gel silica coating demonstrated better durability against spraying damage when compared to tribochemical silica coating.

3. Silica coating prepared by the sol-gel technique showed good biocompatibility.

\section{Acknowledgments}

This work was supported by grants from the National High Technology Research and Development Program of China (863 Program, 2012AA030309), Jiangsu Provincial Department of Education (JH10-27), and the Priority Academic Program Development of Jiangsu Higher Education Institutions (2011-137). The authors thank Wei Huan of the Center for Hygienic Analysis and Detection, Nanjing 
Medical University, for his assistance with biological safety tests.

\section{Disclosure}

The authors report no conflicts of interest in this work.

\section{References}

1. Giordano R, Sabrosa CE. Zirconia: material background and clinical application. Compend Contin Educ Dent. 2010;31(9):710-715.

2. Vagkopoulou T, Koutayas SO, Koidis P, Strub JR. Zirconia in dentistry: Part 1. Discovering the nature of an upcoming bioceramic. Eur J Esthet Dent. 2009;4(2):130-151.

3. Manicone PF, Rossi Iommetti P, Raffaelli L. An overview of zirconia ceramics: basic properties and clinical applications. J Dent. 2007; 35(11):819-826.

4. Wang G, Liu X, Zreiqat H, Ding C. Enhanced effects of nano-scale topography on the bioactivity and osteoblast behaviors of micron rough ZrO2 coatings. Colloids Surf B Biointerfaces. 2011;86(2):267-274.

5. Mair L, Padipatvuthikul P. Variables related to materials and preparing for bond strength testing irrespective of the test protocol. Dent Mater. 2010;26(2):e17-e23.

6. Thompson JY, Stoner BR, Piascik JR, Smith R. Adhesion/cementation to zirconia and other non-silicate ceramics: where are we now? Dent Mater. 2011;27(1):71-82.

7. Lung CY, Matinlinna JP. Aspects of silane coupling agents and surface conditioning in dentistry: an overview. Dent Mater. 2012;28(5): 467-477.

8. Oguri T, Tamaki Y, Hotta Y, Miyazaki T. Effects of a convenient silica-coating treatment on shear bond strengths of porcelain veneers on zirconia-based ceramics. Dent Mater J. 2012;31(5):788-796.

9. Yamaguchi H, Ino S, Hamano N, Okada S, Teranaka T. Examination of bond strength and mechanical properties of Y-TZP zirconia ceramics with different surface modifications. Dent Mater J. 2012;31(3): $472-480$.

10. Chen C, Kleverlaan CJ, Feilzer AJ. Effect of an experimental zirconiasilica coating technique on micro tensile bond strength of zirconia in different priming conditions. Dent Mater. 2012;28(8):e127-e134.

11. Derand T, Molin M, Kvam K. Bond strength of composite luting cement to zirconia ceramic surfaces. Dent Mater. 2005;21(12):1158-1162.
12. Piascik JR, Swift EJ, Thompson JY, Grego S, Stoner BR. Surface modification for enhanced silanation of zirconia ceramics. Dent Mater. 2009;25(9):1116-1121.

13. de Castro HL, Corazza PH, Paes-Júnior Tde A, Della Bona A. Influence of Y-TZP ceramic treatment and different resin cements on bond strength to dentin. Dent Mater. 2012;28(11):1191-1197.

14. Cristoforides P, Amaral R, May LG, Bottino MA, Valandro LF. Composite resin to yttria stabilized tetragonal zirconia polycrystal bonding: comparison of repair methods. Oper Dent. 2012;37(3): 263-271.

15. Lin J, Shinya A, Gomi H, Shinya A. Effect of self-adhesive resin cement and tribochemical treatment on bond strength to zirconia. Int J Oral Sci. 2010;2(1):28-34.

16. Kosmac T, Oblak C, Jevnikar P, Funduk N, Marion L. Strength and reliability of surface treated Y-TZP dental ceramics. J Biomed Mater Res. 2000;53(4):304-313

17. Re D, Augusti D, Augusti G, Giovannetti A. Early bond strength to low-pressure sandblasted zirconia: evaluation of a self-adhesive cement. Eur J Esthet Dent. 2012;7(2):164-175.

18. Kosmac T, Oblak C, Jevnikar P, Funduk N, Marion L. The effect of surface grinding and sandblasting on flexural strength and reliability of Y-TZP zirconia ceramic. Dent Mater. 1999;15(6):426-433.

19. Xie H, Wang X, Wang Y, Zhang F, Chen C, Xia Y. Effects of sol-gel processed silica coating on bond strength of resin cements to glassinfiltrated alumina ceramic. $J$ Adhes Dent. 2009;11(1):49-55.

20. Xie H, Zhu Y, Chen C, Gu N, Zhang F. Effect of silica coating on fracture strength of glass-infiltrated alumina ceramic cemented to dentin. J Adhes Dent. 2011;13(5):467-472.

21. Nishigawa G, Maruo Y, Irie M, et al. Ultrasonic cleaning of silicacoated zirconia influences bond strength between zirconia and resin luting material. Dent Mater J. 2008;27(6):842-848.

22. Valandro LF, Ozcan M, Bottino MC, Bottino MA, Scotti R, Bona AD. Bond strength of a resin cement to high-alumina and zirconia-reinforced ceramics: the effect of surface conditioning. J Adhes Dent. 2006;8(3): 175-181.

23. Fardad MA. Catalysts and the structure of $\mathrm{SiO}_{2}$ sol-gel films. Journal of Materials Science. 2000;35(7):1835-1841.

24. Chen L, Suh BI, Kim J, Tay FR. Evaluation of silica-coating techniques for zirconia bonding. Am J Dent. 2011;24(2):79-84.
International Journal of Nanomedicine

\section{Publish your work in this journal}

The International Journal of Nanomedicine is an international, peerreviewed journal focusing on the application of nanotechnology in diagnostics, therapeutics, and drug delivery systems throughout the biomedical field. This journal is indexed on PubMed Central, MedLine, CAS, SciSearch ${ }^{\circledR}$, Current Contents ${ }^{\circledR} /$ Clinical Medicine,

\section{Dovepress}

Journal Citation Reports/Science Edition, EMBase, Scopus and the Elsevier Bibliographic databases. The manuscript management system is completely online and includes a very quick and fair peer-review system, which is all easy to use. Visit http://www.dovepress.com/ testimonials.php to read real quotes from published authors. 